Mini-review

\title{
An updated meta-analysis to assess the effectiveness of psychological interventions delivered by psychological specialists and generalist clinicians on glycaemic control and on psychological status
}

\author{
Rahul Alam ${ }^{\mathrm{a}, *}$, Jackie Sturt ${ }^{\mathrm{a}}$, Ranjit Lall ${ }^{\mathrm{b}}$, Kirsty Winkley ${ }^{\mathrm{c}}$ \\ ${ }^{a}$ Health Sciences Research Institute, Warwick Medical School, University of Warwick, Gibbet Hill, Coventry CV4 7AL, UK \\ ${ }^{\mathrm{b}}$ Clinical Trials Unit, Warwick Medical School, UK \\ ${ }^{\mathrm{C}}$ Academic Department of Psychological Medicine, Institute of Psychiatry, UK
}

\section{A R T I C L E I N F O}

\section{Article history:}

Received 9 June 2008

Received in revised form 11 August 2008

Accepted 30 August 2008

Available online $\mathrm{xxx}$

\section{Keywords:}

Diabetes

Psychological interventions

Glycaemic control

$\mathrm{HbA}_{1 \mathrm{c}}$

Psychological specialist

Generalist clinicians

Health care delivery

\begin{abstract}
A B S T R A C T
Objective: To update a meta-analysis and determine the effectiveness of psychological interventions on glycaemic control measured by $\mathrm{HbA}_{1 \mathrm{c}}$ and psychological status in type 2 diabetes and to compare effects when interventions are delivered by generalist clinicians compared to psychological specialists. Methods: We used the original review protocol and searched the Cochrane central register of controlled trials, Medline, Embase, PsychLIT, and Google Scholar from February 2003 (end of previous review) to March 2007. We extracted data on the participants, interventions, delivery methods, comparison groups and outcome measures.

Results: 35 trials were reviewed and meta-analysis of 19 trials $(n=1431)$, reporting $\mathrm{HbA}_{1 \mathrm{c}}$ found a reduction in $\mathrm{HbA}_{1 \mathrm{c}}$ by $0.54 \%(-0.32 ; 95 \% \mathrm{CI}$ : -0.47 to -0.16$)$. In nine trials $(n=832)$ interventions were delivered by diabetes or general clinicians reducing $\mathrm{HbA}_{1 \mathrm{c}}$ by $0.51 \%(-0.27 ; 95 \% \mathrm{CI}:-0.50$ to 0.04$)$. In nine trials, interventions $(n=561)$ were delivered by psychological specialists reducing $\mathrm{HbA}_{1 \mathrm{c}}$ by $0.57 \%$ $(-0.36$; $95 \% \mathrm{CI}$ : -0.61 to 0.12$)$. Meta-analysis of 13 trials reporting psychological status found psychological status to be lower in the intervention groups -0.56 ( $95 \% \mathrm{CI}: 1.00$ to -0.13 ). Trial quality for the majority of studies remained poor.

Conclusion: Our findings suggest that psychological and general clinicians are similarly effective in delivering psychological interventions, however, effect sizes for all clinicians have reduced since the earlier review.

Practice implications: Psychological training opportunities for generalist clinicians could lead to wider availability of effective psychological care.
\end{abstract}

(c) 2008 Elsevier Ireland Ltd. All rights reserved.

\section{Introduction}

\subsection{Background}

Diabetes is a complex metabolic condition in which the patient has a life-long responsibility for managing their condition. The first aim of diabetes management is the control of blood glucose levels, usually termed glycaemic control, which is measured by an $\mathrm{HbA}_{1 \mathrm{c}}$ blood test. $\mathrm{HbA}_{1 \mathrm{c}}$ measures the amount of circulating glucose in

\footnotetext{
* Corresponding author. Tel.: +44 2476 574490; fax: +44 2476528375.

E-mail address: R.Alam@warwick.ac.uk (R. Alam).

Abbreviations: $\mathrm{HbA}_{1 \mathrm{c}}$, glycaemic control; $\mathrm{CBT}$, cognitive behaviour therapy; RCTs, randomised controlled trials; GPs, general practitioners; $I^{2}$, Higgins $I$ squared test; NS, not stated; D, diet; T, tablets; I, insulin; TTM, transtheoretical model of change.
}

the blood over a 120-day period. The aim of treatment and patient management is to achieve $\mathrm{HbA}_{1 \mathrm{c}}$ levels $\leq 6.5 \%$ and the UKPDS [1] established that every $1 \%$ reduction in $\mathrm{HbA}_{1 \mathrm{c}}$ towards this goal carries a $14-37 \%$ reduction in serious diabetes-related complications such as heart disease, blindness and kidney disease [1]. In order to achieve optimum glycaemic control people living with diabetes have to regulate their behaviour on a daily basis. The behavioural challenges imposed by close attention to diet, medication regimens including subcutaneous insulin injections, exercise, weight management, self-monitoring of daily blood glucose levels and foot care are burdensome and have social and emotional consequences [2]. In addition to this, specific psychological problems including depressive disorders $[3,4]$ and eating disorders [5] can manifest themselves through poor glycaemic control thus giving rise to further complications. 


\subsection{Previous review}

Ismail et al. [2] published a systematic review and metaanalysis in 2004 which identified the potential clinical and psychological benefits of offering psychological interventions to patients with type 2 diabetes. The review [2] incorporated interventions comprising the most commonly used psychotherapeutic models used in health-care settings. They included supportive or counselling therapy (including motivational interviewing and non-directive counselling), brief psychodynamic therapy, interpersonal psychotherapy and cognitive behaviour therapy and its associated techniques including contract setting, goal setting, problem solving, activity scheduling, stress management and relaxation.

Ismail et al. [2] identified 25 trials and observed significant reductions in $\mathrm{HbA}_{1 \mathrm{c}}$ of $1 \%$, similar to that of the UKPDS [1] but notably and unlike the UKPDS, these were achieved by nonpharmacological means. Psychological distress was also reduced. The findings suggest that psychological therapies may be a useful adjunct to current routine diabetes care.

However, despite the apparent benefits of psychological care in diabetes [6-8], there remains a severe shortage of psychological specialists within mainstream health care preventing access for the majority of patients [9]. Ismail et al.'s [2] review included a number of trials in which the psychological intervention had been delivered by a physician or nurse (generalist clinicians) compared to delivery by a professional with specialist training in psychology (specialist). It is not clear whether the discipline and training of the therapist makes a difference to outcome. If psychological interventions can be effectively delivered by generalist clinicians, access to this care becomes more possible for patients.

\subsection{Goals of the current study}

This paper reports an update to the Ismail et al. [2] review and meta-analysis to establish whether the $1 \%$ reduction in $\mathrm{HbA}_{1 \mathrm{c}}$ has been maintained and to compare the impact of the intervention on glycaemic control and psychological status if the intervention is delivered by generalist clinicians compared to psychological specialists. Additionally, the previous review was subjected to some criticism upon publication and there was concern that the small sample sizes of included trials and poor trial quality had resulted in an overestimation of treatment effect [10,11]. We will report on whether the quality of psychological intervention trials has improved subsequent to the more recent common acceptance of the CONSORT trial quality criteria [12].

\section{Methods}

\subsection{Selection criteria}

The review follows the original protocol [13] and eligible studies were randomised control trials (RCT's) utilising a psychological intervention in adults with type 2 diabetes. Studies describing psychological therapies were included as were psychoeducational studies with a detailed psychological component and where the intervention balance was more towards the psychological component. We classified the interventions based on the psychological techniques used, mode of delivery and type of control employed. Interventions not clearly describing the psychological components were omitted.

Specialist psychological therapists were defined as professionals whose speciality was in the psychological care of people and included psychologists, psychiatrists, psychotherapists and counsellors. Generalist clinicians were defined as professionals whose speciality was in the provision of general and specialist diabetes clinical care and included GPs, diabetes physicians, nurses, dieticians and occupational therapists. Outcome measures were glycaemic control measured by $\mathrm{HbA}_{1 \mathrm{c}}$ and measures of psychological status (anxiety and/or depression).

\subsection{Search strategy}

Using the Cochrane collaboration's optimum search strategy for randomised controlled trials the search co-ordinator from the earlier review updated the search from February 2003 to March 2007. Details of the search strategy are published elsewhere [13].

\subsection{Study selection}

Two authors (JS \& KW) independently assessed the abstracts for inclusion in the review and inter-rater reliability was reported using Cohen's kappa $(\kappa)$ [14]. We included abstracts of papers where a controlled trial of a psychological intervention for type 2 diabetes was described. Where differences between raters, or ambiguity occurred, the full papers were retrieved and disagreements were resolved through dialogue. Three authors (JS, RA and $\mathrm{KW}$ ) independently reviewed the full text papers for inclusion.

\subsection{Data extraction}

JS \& RA independently extracted the data of included studies using the original extraction form. Papers written in languages other than English underwent data-extraction by a native-speaking-registered translator and RA. For studies comparing more than one intervention group we included the most intense psychologically underpinned intervention for analysis.

We recorded age of participants, numbers at baseline and follow-up, study country of origin and setting (including the format, type and duration of intervention), delivery specialist, results and we assessed the study quality. Where data was missing, we contacted the original authors for clarification in order to include in the review.

\subsection{Statistical analysis}

Data was entered into SPSS (version 14.0) and the metaanalysis was carried out using the metan command in STATA (intercooled version 9.0).

\subsubsection{Meta-analysis and heterogeneity}

When combining data in meta-analysis, no two studies are likely to be identical and heterogeneity is the variation that exists between the individual studies.

Clinical heterogeneity is described as variability amongst the participants, the interventions and study outcomes measures. Methodological heterogeneity is described as the variability in study design and risk of bias. Statistical heterogeneity is the consequence of clinical or methodological heterogeneity or both and we refer to this simply as heterogeneity. If the clinical and methodological heterogeneity was considered minimal in the studies, then a meta-analysis was deemed possible. The method for detecting heterogeneity in the studies was planned through (a) observation of the forest plot to examine how well the confidence intervals overlay; (b) performance of a Chi-squared $\left(\chi^{2}\right)$ test with a $p$-value of $>0.10$ [15] and (c) by quantifying the effect of heterogeneity using $I^{2}$ [16]. A small $p$-value from the $\chi^{2}$ test is used to indicate evidence of heterogeneity. However, the statistical power of this test is limited in the presence of few studies. For this reason, a $p$-value of less than 0.10 is often used to indicate 
heterogeneity rather than the conventional cut-off point of $p=0.05$. The $I^{2}$ test is a useful adjunct to assess heterogeneity and provides a quantified measure of consistency between the trials, where values of $25 \%, 50 \%$ and $75 \%$ represent low, moderate and high levels of heterogeneity respectively [16].

\subsubsection{Meta-analytical procedures}

Meta-analysis was deemed appropriate if there were five or more trials with minimal heterogeneity for inclusion which measure the same outcome. The two outcome measures used in our meta-analysis were $\mathrm{HbA}_{\mathrm{lc}}$ and measures of psychological distress.

The mean and standard deviation at baseline and follow-up were extracted for each intervention and each outcome. The mean change from baseline (follow-up-baseline) was extracted for each outcome and this provided the within-group mean. The pooled standard deviations for the mean change scores were obtained and provided the within-group standard deviation. If this standard deviation was missing, then the square root of the baseline and follow-up variance was obtained. This approach is based on the assumption that the correlation between the baseline and followup outcome values is 0.5 .

\subsubsection{Effect size}

The difference in the mean change values between the two interventions (treatment-control) divided by the pooled standard deviation for this difference provided the estimate of the effect size. This was obtained for each assessment, which was then used in the meta-analysis. The effect sizes or the standardised mean difference method expresses the size of the treatment in each trial relative to the variability observed in that trial. The method allows different measures of the same outcome to be combined because different methods for measuring the outcomes were used.

\subsubsection{Random effect and fixed effect models}

In order to obtain a common effect across all the studies, fixed effects and random effects meta-analysis models were computed and the results compared. With fixed effects models all of the studies that are examined as a whole are considered to have been conducted under similar conditions with similar subjects. The random effects models generally produce wider confidence intervals and allow the study outcomes to vary in a normal distribution between studies. As with the original review [2] we anticipated some heterogeneity and use of the random effects model enabled us to minimise the impact of "background noise" presented by individual studies.

\subsubsection{Transformation of effect sizes}

For the outcome of $\mathrm{HbA}_{1 \mathrm{c}}$ we converted the estimated pooled standardised effect size into absolute units by multiplying the estimate by the pooled S.D. of all the studies included in the metaanalysis for each outcome. In the case where different studies gave different results, reasons why effects differed across studies were assessed using meta-regression analysis (using metareg in STATA (version 9)). Both these techniques are used to see if particular characteristics of studies are related to the sizes of the treatment effect. The number of sessions and duration of therapy, which are deemed proxy measures of intensity and duration of follow-up were used as potential covariates in a regression meta-analysis.

\subsection{Quality assessment}

Quality assessment criteria developed by Schulz et al. [17] and Jadad et al. [18] was used to assess for selection, attrition and detection bias.

\subsection{Publication bias}

Publication bias is the phenomenon in which (i) positive results have a better chance of being published, (ii) are published in journals with higher impact factors (and are more likely to be retrieved in medical literature searches) and (iii) are published faster. Publication bias was estimated by using a funnel plot for the treatment effect (using the treatment difference) and its standard error [19]. In the absence of publication bias, we would expect less precise studies (small sample size, duration, follow-up) to be more affected by chance alone, thus widely scattered from the pooled estimate. As studies get larger with more events, we expect them to be closer to the pooled estimate with a resulting triangular shape (or funnel), split by a line representing the pooled estimate of the meta-analysis. Formally, the Begg's adjusted rank correlation test was used to assess if there is a significant correlation between the standardised effect estimates and their variances [20].

\subsection{Sensitivity analysis}

Sensitivity analysis was performed using fixed and random effects models, by excluding borderline studies, by excluding studies using a less intensive psychological therapy as controls, by excluding studies with clinical sub-groups and by comparing poor quality studies with the higher quality studies.

\subsection{Role of the funding source}

The funders of the study had no involvement with any aspect of the study, including the decision to submit this report for publication.

\section{Results}

\subsection{Description of new trials}

The 2003-2007 searches identified 3218 new studies from which 48 full texts were selected for further extraction with a substantial level of agreement between the reviewers $(\kappa=0.67)$ [14]. Fig. 1 shows the flow of original and newly identified studies considered for the review. Ten new trials which were published after the CONSORT statement [12] were identified for inclusion in the systematic review.

\subsubsection{Study quality}

One study was rated as quality B [21] and nine studies rated as C [22-30].

\subsubsection{Psychological therapies used}

Seven studies utilised cognitive behaviour therapy [22-28], two trials utilised counselling techniques $[29,30]$ and one trial utilised psychotherapy [21].

\subsubsection{Intervention delivery specialist}

Five of these trials were delivered by generalists $[23,25,26,30,31]$, three were delivered by psychological specialists $[21,24,27]$ and two did not report the specialist $[22,28]$.

\subsubsection{Intervention format}

The mean intervention duration was 18.3 (S.D. 11.9) weeks. The majority of studies were delivered in a $1: 1$ format $[22,23,25,26-$ $28,30,31]$ and two were delivered in a combined group and 1:1 format $[21,24]$. 
Characteristics of studies included in the systematic review of randomised controlled trials incorporating psychological interventions for patients with type 2 diabetes

\begin{tabular}{|c|c|c|c|c|c|c|c|c|c|c|c|}
\hline $\begin{array}{l}\text { Year, country, } \\
\text { reference }\end{array}$ & $\begin{array}{l}\text { Number of } \\
\text { participants } \\
\text { recruited/at } \\
\text { follow-up }\end{array}$ & $\begin{array}{l}\text { Mean } \\
\text { age } \\
\text { (S.D. or } \\
\text { range) } \\
\text { (years) }\end{array}$ & $\begin{array}{l}\text { Mean (S.D.) } \\
\% \text { glycated } \\
\text { haemoglo- } \\
\text { bin at base- } \\
\text { line }\end{array}$ & $\begin{array}{l}\text { Clinical sub- } \\
\text { group (type of } \\
\text { treatment) }\end{array}$ & $\begin{array}{l}\text { Mean (S.D. } \\
\text { or range) } \\
\text { duration of } \\
\text { diabetes } \\
\text { (years) }\end{array}$ & $\begin{array}{l}\text { Model and duration } \\
\text { of therapy in inter- } \\
\text { vention group }\end{array}$ & $\begin{array}{l}\text { Regimen in } \\
\text { intervention group and } \\
\text { speciality of therapist }\end{array}$ & $\begin{array}{l}\text { Model and duration } \\
\text { of therapy in } \\
\text { control group }\end{array}$ & $\begin{array}{l}\text { Regimen in control group } \\
\text { and speciality of therapist }\end{array}$ & $\begin{array}{l}\text { Follow-up } \\
\text { months }\end{array}$ & Quality \\
\hline $\begin{array}{l}\text { 1983, } \\
\text { Canada, } \\
\text { Rabkin }\end{array}$ & $40 / 40$ & $\begin{array}{l}53-9 \\
(2-0)\end{array}$ & NS & $\begin{array}{l}\text { Sub-optimum } \\
\text { glycaemic } \\
\text { control (D, T) }\end{array}$ & NS & $\begin{array}{l}\text { Group CBT for } 6 \\
\text { weeks }\end{array}$ & $\begin{array}{l}6 \text { behaviour } \\
\text { modification sessions } \\
\text { by nutritionist }\end{array}$ & $\begin{array}{l}\text { Individual } \\
\text { education for } 6 \\
\text { weeks }\end{array}$ & $\begin{array}{l}2 \text { individual dietary } \\
\text { counselling sessions by } \\
\text { nutritionist }\end{array}$ & 3 & C \\
\hline $\begin{array}{l}\text { 1985, USA, } \\
\text { Wing }\end{array}$ & $53 / 50$ & $\begin{array}{l}55-1 \\
(7-3)\end{array}$ & $9.33(0.3)$ & Obese $(D, T)$ & 5-9 (NS) & $\begin{array}{l}\text { Group CBT for } 16 \\
\text { weeks }\end{array}$ & $\begin{array}{l}16 \text { behaviour } \\
\text { modification sessions } \\
\text { by psychologist and } \\
\text { nutritionist }\end{array}$ & $\begin{array}{l}\text { Group education } \\
\text { for } 16 \text { weeks }\end{array}$ & $\begin{array}{l}4 \text { standard education } \\
\text { sessions by psychologist } \\
\text { and nutritionist }\end{array}$ & 4 & c \\
\hline $\begin{array}{r}\text { 1986, USA, } \\
\text { Hartwell }\end{array}$ & $76 / 76$ & NS & $8.7(2.7)$ & $\begin{array}{l}\text { Sub-optimum } \\
\text { glycaemic } \\
\text { control } \\
\text { (D, T) }\end{array}$ & NS & $\begin{array}{l}\text { Group CBT for } 10 \\
\text { weeks }\end{array}$ & $\begin{array}{l}14 \mathrm{CBT} \text { sessions by } \\
\text { dietician and exercise } \\
\text { leader }\end{array}$ & $\begin{array}{l}\text { Group education } \\
\text { for } 10 \text { weeks }\end{array}$ & $\begin{array}{l}10 \text { traditional education } \\
\text { sessions by } \\
\text { multidisciplinary diabetes } \\
\text { team }\end{array}$ & 6 & c \\
\hline $\begin{array}{l}\text { 1986, USA, } \\
\text { White }\end{array}$ & $41 / 32$ & $\begin{array}{l}61-6 \\
(6-4)\end{array}$ & $11.3(3.1)$ & $\begin{array}{l}\text { Men, obese, } \\
\text { sub-optimum } \\
\text { glycaemic } \\
\text { control (D, T, I) }\end{array}$ & $\begin{array}{l}12-6 \\
(11-4)\end{array}$ & $\begin{array}{l}\text { Group CBT for } 6 \\
\text { months }\end{array}$ & $\begin{array}{l}16 \text { group management } \\
\text { sessions by } \\
\text { psychologist }\end{array}$ & $\begin{array}{l}\text { Group education } \\
\text { for } 6 \text { months }\end{array}$ & $\begin{array}{l}16 \text { advice/education } \\
\text { sessions by nurse and } \\
\text { dietician }\end{array}$ & 6 & C \\
\hline $\begin{array}{l}\text { 1987, USA, } \\
\text { Heitzmann }\end{array}$ & $46 / 46$ & $\begin{array}{l}52-9 \\
(12-1)\end{array}$ & $11.3(2.3)$ & General (D, T, I) & NS & $\begin{array}{l}\text { Group and } \\
\text { individual CBT for } 7 \\
\text { weeks }\end{array}$ & $\begin{array}{l}7 \text { group and } 9 \\
\text { individual CBT } \\
\text { sessions; speciality not } \\
\text { specified }\end{array}$ & $\begin{array}{l}\text { Group CBT } \\
\text { (duration not } \\
\text { specified) }\end{array}$ & $\begin{array}{l}\text { Minimal relaxation } \\
\text { training: speciality not } \\
\text { specified }\end{array}$ & $6^{*}$ & B \\
\hline $\begin{array}{r}\text { 1987, Spain, } \\
\text { Rodriguez }\end{array}$ & $11 / 11$ & $\begin{array}{l}60-7 \\
(51- \\
70)\end{array}$ & NS & $\begin{array}{l}\text { General } \\
\text { (treatments not } \\
\text { specified) }\end{array}$ & $4-3(3-7)$ & $\begin{array}{l}\text { Individual CBT and } \\
\text { education for } 5 \\
\text { weeks }\end{array}$ & $\begin{array}{l}3 \text { CBT sessions and } 2 \\
\text { education sessions by } \\
\text { psychologist }\end{array}$ & $\begin{array}{l}\text { Individual } \\
\text { education for } 5 \\
\text { weeks }\end{array}$ & $\begin{array}{l}2 \text { education sessions; } \\
\text { speciality not specified }\end{array}$ & 2 & C \\
\hline $\begin{array}{l}\text { 1990, } \\
\text { Australia, } \\
\text { Campbell }\end{array}$ & $62 / 61$ & $\begin{array}{l}58-5 \\
(9-0)\end{array}$ & $11.6(1.9)$ & $\begin{array}{l}\text { Sub-optimum } \\
\text { glycaemic } \\
\text { control obese } \\
(\mathrm{D}, \mathrm{T}, \mathrm{I})\end{array}$ & $7-5(7-0)$ & $\begin{array}{l}\text { Group counselling } \\
\text { for } 11 \text { weeks }\end{array}$ & $\begin{array}{l}\text { Cognitive motivational } \\
\text { therapy (number of } \\
\text { sessions NS) by } \\
\text { physician, dietician and } \\
\text { psychologist }\end{array}$ & $\begin{array}{l}\text { Group education } \\
\text { (duration not } \\
\text { specified) }\end{array}$ & $\begin{array}{l}3 \text { education sessions; } \\
\text { speciality not specified }\end{array}$ & 6 & B \\
\hline $\begin{array}{l}\text { 1991, USA, } \\
\text { Wing }\end{array}$ & $49 / 43$ & $\begin{array}{l}52-5 \\
(7-5)\end{array}$ & $9.9(2.2)$ & Obese (D, T, I) & NS & $\begin{array}{l}\text { Group CBT and } \\
\text { couple therapy for } \\
10 \text { weeks }\end{array}$ & $\begin{array}{l}16 \text { behavioural } \\
\text { modification and } \\
\text { couple therapy sessions } \\
\text { by multidisciplinary } \\
\text { team (project physician } \\
\text { and trained nutritionist } \\
\text { reported) }\end{array}$ & $\begin{array}{l}\text { Group CBT for } 10 \\
\text { weeks }\end{array}$ & $\begin{array}{l}16 \text { behavioural } \\
\text { modification sessions by } \\
\text { multidisciplinary team }\end{array}$ & 5 & c \\
\hline $\begin{array}{l}\text { 1992, USA, } \\
\text { D'Eramo }\end{array}$ & $54 / 34$ & $\begin{array}{l}56-0 \\
(8-1)\end{array}$ & $11.1(2.8)$ & Obese (D, T) & NS & $\begin{array}{l}\text { Group education } \\
\text { and individual } \\
\text { counselling for } 11 \\
\text { weeks }\end{array}$ & $\begin{array}{l}11 \text { intensive group } \\
\text { education and } 2 \\
\text { individual counselling } \\
\text { sessions by nurse }\end{array}$ & $\begin{array}{l}\text { Education for } 1 \\
\text { week }\end{array}$ & $\begin{array}{l}1 \text { minimal education } \\
\text { session by nurse }\end{array}$ & 6 & c \\
\hline $\begin{array}{l}\text { 1993, USA, } \\
\text { Boehm }\end{array}$ & $156 / 135$ & $\begin{array}{l}58-0 \\
(11-3)\end{array}$ & NS & General (D, T, I) & NS & $\begin{array}{l}\text { Individual CBT } \\
\text { (duration not } \\
\text { specified) }\end{array}$ & $\begin{array}{l}\text { Behaviour modification } \\
\text { delivered by clinical } \\
\text { nurse specialist } \\
\text { (number of sessions } \\
\text { NS) }\end{array}$ & Usual care & $\begin{array}{l}\text { Regimen not described; } \\
\text { speciality not specified }\end{array}$ & NS & C \\
\hline $\begin{array}{l}\text { 1993, USA, } \\
\text { Lane }\end{array}$ & $38 / 38$ & NS & $10.3(2.3)$ & $\begin{array}{l}\text { Sub-optimum } \\
\text { glycaemic } \\
\text { control (D, T) }\end{array}$ & NS & $\begin{array}{l}\text { Individual CBT for } 8 \\
\text { weeks and } \\
\text { intensive diabetes } \\
\text { care for } 12 \text { months }\end{array}$ & $\begin{array}{l}8 \text { relaxation training } \\
\text { sessions and intensive } \\
\text { diabetes care; } \\
\text { speciality not specified }\end{array}$ & $\begin{array}{l}\text { Intensive diabetes } \\
\text { care for } 12 \text { months }\end{array}$ & $\begin{array}{l}\text { Intensive diabetes } \\
\text { education and clinical care; } \\
\text { speciality not specified }\end{array}$ & 6 & B \\
\hline $\begin{array}{l}\text { 1996, } \\
\text { Australia, } \\
\text { Campbell }\end{array}$ & $118 / 60$ & $\begin{array}{l}59-5 \\
(10-2)\end{array}$ & $12.6(4.3)$ & General (D, T) & $0-4(0-1)$ & $\begin{array}{l}\text { Individual CBT for } \\
12 \text { months }\end{array}$ & $\begin{array}{l}6 \text { CBT sessions by nurse } \\
\text { educator }\end{array}$ & $\begin{array}{l}\text { Group education } \\
\text { for } 2 \text { weeks }\end{array}$ & $\begin{array}{l}2 \text { minimal education } \\
\text { sessions by nurse educator } \\
\text { and dietician }\end{array}$ & 6 & B \\
\hline
\end{tabular}


Table 1 (Continued)

\begin{tabular}{|c|c|c|c|c|c|c|c|c|c|c|c|}
\hline $\begin{array}{l}\text { Year, country, } \\
\text { reference }\end{array}$ & $\begin{array}{l}\text { Number of } \\
\text { participants } \\
\text { recruited/at } \\
\text { follow-up }\end{array}$ & $\begin{array}{l}\text { Mean } \\
\text { age } \\
\text { (S.D. or } \\
\text { range) } \\
\text { (years) }\end{array}$ & $\begin{array}{l}\text { Mean (S.D.) } \\
\% \text { glycated } \\
\text { haemoglo- } \\
\text { bin at base- } \\
\text { line }\end{array}$ & $\begin{array}{l}\text { Clinical sub- } \\
\text { group (type of } \\
\text { treatment) }\end{array}$ & $\begin{array}{l}\text { Mean (S.D. } \\
\text { or range) } \\
\text { duration of } \\
\text { diabetes } \\
\text { (years) }\end{array}$ & $\begin{array}{l}\text { Model and duration } \\
\text { of therapy in inter- } \\
\text { vention group }\end{array}$ & $\begin{array}{l}\text { Regimen in } \\
\text { intervention group and } \\
\text { speciality of therapist }\end{array}$ & $\begin{array}{l}\text { Model and duration } \\
\text { of therapy in } \\
\text { control group }\end{array}$ & $\begin{array}{l}\text { Regimen in control group } \\
\text { and speciality of therapist }\end{array}$ & $\begin{array}{l}\text { Follow-up } \\
\text { months }\end{array}$ & Quality \\
\hline $\begin{array}{l}\text { 1997, USA, } \\
\text { Aikens }\end{array}$ & $22 / 22$ & $\begin{array}{l}61-0 \\
(10-2)\end{array}$ & $11.0(1.9)$ & General (D, T, I) & $11-0(9-0)$ & $\begin{array}{l}\text { Group CBT for } 8 \\
\text { weeks }\end{array}$ & $\begin{array}{l}6 \text { relaxation training } \\
\text { sessions by } \\
\text { psychologist }\end{array}$ & Usual care & $\begin{array}{l}\text { Routine medical care; } \\
\text { speciality not specified }\end{array}$ & 4 & c \\
\hline $\begin{array}{l}\text { 1997, } \\
\text { Australia, } \\
\text { Henry }\end{array}$ & $19 / 19$ & $\begin{array}{l}60-0 \\
(47- \\
74)\end{array}$ & $10.7(1.9)$ & $\begin{array}{l}\text { Sub-optimum } \\
\text { glycaemic } \\
\text { control, stress/ } \\
\text { anxiety (D, T, I) }\end{array}$ & $\begin{array}{l}6-4(1-5- \\
23-0)\end{array}$ & $\begin{array}{l}\text { Group CBT for } 6 \\
\text { weeks }\end{array}$ & $\begin{array}{l}6 \text { stress management } \\
\text { sessions by } \\
\text { psychologist }\end{array}$ & Waiting list & Usual practice by physician & $2-25$ & c \\
\hline $\begin{array}{l}\text { 1997, USA, } \\
\text { Jablon }\end{array}$ & $20 / 20$ & $\begin{array}{l}58-9 \\
(7-7)\end{array}$ & $6.5(1.8)$ & $\begin{array}{l}\text { Sub-optimum } \\
\text { glycaemic } \\
\text { control }(\mathrm{D}, \mathrm{T})\end{array}$ & $7-9(8-7)$ & $\begin{array}{l}\text { Individual CBT for } 4 \\
\text { weeks }\end{array}$ & $\begin{array}{l}8 \text { relaxation therapy } \\
\text { sessions by psycho- } \\
\text { physiologist }\end{array}$ & Waiting list & $\begin{array}{l}\text { None; speciality not } \\
\text { specified }\end{array}$ & 1 & C \\
\hline $\begin{array}{l}\text { 1997, USA, } \\
\text { Smith }\end{array}$ & $22 / 16$ & $\begin{array}{l}62-4 \\
(7-0)\end{array}$ & $10.3(2.2)$ & $\begin{array}{l}\text { Women, obese } \\
(\mathrm{D}, \mathrm{T})\end{array}$ & $6-7(5-4)$ & $\begin{array}{l}\text { Group CBT and } \\
\text { individual } \\
\text { counselling for } 4 \\
\text { months }\end{array}$ & $\begin{array}{l}16 \text { group behaviour } \\
\text { modification by } \\
\text { nutritionist, exercise } \\
\text { psychologist, and } \\
\text { psychologist and } 3 \\
\text { individual sessions by } \\
\text { psychologist. }\end{array}$ & $\begin{array}{l}\text { Group CBT for } 4 \\
\text { months }\end{array}$ & $\begin{array}{l}16 \text { group behaviour } \\
\text { modification by } \\
\text { nutritionist, exercise } \\
\text { psychologist, and } \\
\text { psychologist }\end{array}$ & 4 & c \\
\hline $\begin{array}{l}\text { 1998, USA, } \\
\text { Lustman }\end{array}$ & $51 / 42$ & $\begin{array}{l}54-8 \\
(10-1)\end{array}$ & $10.3(3.4)$ & $\begin{array}{l}\text { Depression } \\
(\mathrm{D}, \mathrm{T}, \mathrm{I})\end{array}$ & $8-8(9-5)$ & $\begin{array}{l}\text { Group CBT and } \\
\text { education for } 10 \\
\text { weeks }\end{array}$ & $\begin{array}{l}30 \text { CBT sessions by } \\
\text { psychologist and } 20 \\
\text { education sessions by } \\
\text { diabetes educator }\end{array}$ & $\begin{array}{l}\text { Group education } \\
\text { for } 10 \text { weeks }\end{array}$ & $\begin{array}{l}20 \text { education sessions by } \\
\text { diabetes educator }\end{array}$ & $6^{*}$ & A \\
\hline $\begin{array}{l}\text { 1999, USA, } \\
\text { Ridgeway }\end{array}$ & $38 / 38$ & $\begin{array}{l}63-5 \\
\text { NS }\end{array}$ & $12.3(2.6)$ & General (D, T, I) & $11-5(\mathrm{NS})$ & $\begin{array}{l}\text { Group CBT and } \\
\text { education for } 6 \\
\text { months }\end{array}$ & $\begin{array}{l}6 \text { behaviour } \\
\text { modification sessions } \\
\text { and education by } \\
\text { diabetes educators }\end{array}$ & Usual care & $\begin{array}{l}\text { Usual office visits by family } \\
\text { physicians }\end{array}$ & 6 & B \\
\hline $\begin{array}{l}\text { 2001, China, } \\
\text { Huang }\end{array}$ & $59 / 59$ & NS & $9.9(2.1)$ & $\begin{array}{l}\text { Depression } \\
\text { (treatments not } \\
\text { specified) }\end{array}$ & NS & $\begin{array}{l}\text { Group counselling } \\
\text { for } 3 \text { months }\end{array}$ & $\begin{array}{l}24 \text { supportive } \\
\text { psychotherapy sessions } \\
\text { by psychiatrist }\end{array}$ & Usual care & $\begin{array}{l}\text { Conventional diabetes care; } \\
\text { speciality not specified }\end{array}$ & 3 & c \\
\hline $\begin{array}{l}\text { 2001, USA, } \\
\text { McKay }\end{array}$ & $78 / 68$ & $\begin{array}{l}52-3 \\
(\mathrm{NS})\end{array}$ & NS & $\begin{array}{l}\text { Sedentary } \\
(\mathrm{D}, \mathrm{T}, \mathrm{I})\end{array}$ & NS & $\begin{array}{l}\text { Individual CBT for } 8 \\
\text { weeks }\end{array}$ & $\begin{array}{l}\text { Internet-based physical } \\
\text { activity intervention by } \\
\text { occupational therapist }\end{array}$ & $\begin{array}{l}\text { Individual } \\
\text { education } \\
\text { (duration not } \\
\text { specified) }\end{array}$ & $\begin{array}{l}\text { Internet-based education; } \\
\text { speciality not specified }\end{array}$ & 2 & c \\
\hline $\begin{array}{l}\text { 2001, China, } \\
\text { Zhu }\end{array}$ & $59 / 56$ & $\begin{array}{l}57-4 \\
(9-4)\end{array}$ & NS & $\begin{array}{l}\text { General } \\
\text { (treatments not } \\
\text { specified) }\end{array}$ & $7-4(5-4)$ & $\begin{array}{l}\text { Individual CBT for } 1 \\
\text { month }\end{array}$ & $\begin{array}{l}\text { Relaxation training by } \\
\text { psychiatrist (number of } \\
\text { sessions not given) }\end{array}$ & $\begin{array}{l}\text { Usual care } \\
\text { (duration not } \\
\text { specified) }\end{array}$ & $\begin{array}{l}\text { Conventional diabetes care; } \\
\text { speciality not specified }\end{array}$ & 1 & c \\
\hline $\begin{array}{l}\text { 2002, } \\
\text { Australia, } \\
\text { Kenardy }\end{array}$ & $34 / 32$ & $\begin{array}{l}55-0 \\
(10-5)\end{array}$ & $7.5(1.5)$ & $\begin{array}{l}\text { Binge-eating } \\
(\mathrm{D}, \mathrm{T}, \mathrm{I})\end{array}$ & $3-2(5-7)$ & $\begin{array}{l}\text { Group CBT for } 10 \\
\text { weeks }\end{array}$ & $\begin{array}{l}10 \text { CBT sessions by } \\
\text { psychologist }\end{array}$ & $\begin{array}{l}\text { Group counselling } \\
\text { for } 10 \text { weeks }\end{array}$ & $\begin{array}{l}10 \text { non-prescriptive } \\
\text { therapy sessions by } \\
\text { psychologist }\end{array}$ & $2-5$ & B \\
\hline $\begin{array}{l}\text { 2002, USA, } \\
\text { Surwit }\end{array}$ & $108 / 72$ & $\begin{array}{l}57-4 \\
(10-9)\end{array}$ & $7.8(1.8)$ & General $(D, T)$ & NS & $\begin{array}{l}\text { Group CBT and } \\
\text { education for } 2 \\
\text { months }\end{array}$ & $\begin{array}{l}5 \text { education and stress } \\
\text { management training } \\
\text { sessions; speciality not } \\
\text { specified }\end{array}$ & $\begin{array}{l}\text { Group education } \\
\text { for } 2 \text { months }\end{array}$ & $\begin{array}{l}5 \text { education sessions; } \\
\text { speciality not specified }\end{array}$ & 12 & c \\
\hline $\begin{array}{l}\text { 2002, Japan, } \\
\text { Tsujiuchi }\end{array}$ & $36 / 26$ & $\begin{array}{l}62-2 \\
(8-2)\end{array}$ & $8.2(1.7)$ & $\begin{array}{l}\text { General } \\
\text { (treatments not } \\
\text { specified) }\end{array}$ & NS & $\begin{array}{l}\text { Group CBT for } 4 \\
\text { months }\end{array}$ & $\begin{array}{l}16 \text { Qi-gong relaxation } \\
\text { training sessions by } \\
\text { Chinese Qi-gong doctor } \\
\text { (Ph.D. in medicine) }\end{array}$ & Waiting list & $\begin{array}{l}\text { Conventional diabetes care; } \\
\text { speciality not specified }\end{array}$ & 4 & c \\
\hline $\begin{array}{l}\text { 2003, China, } \\
\text { Zeng }\end{array}$ & $108 / 106$ & $\begin{array}{l}56.0 \\
\text { (NS) }\end{array}$ & $10.5(\mathrm{NS})$ & General (D, T, I) & $5.5(2-11)$ & $\begin{array}{l}\text { Group and single } \\
\text { CBT and education } \\
\text { for } 6 \text { months }\end{array}$ & $\begin{array}{l}24 \text { sessions ( } 30- \\
60 \text { min) of supportive } \\
\text { psychotherapy, } \\
\text { relaxation and } \\
\text { education Combination } \\
\text { of psychotherapist \& } \\
\text { clinician }\end{array}$ & $\begin{array}{l}\text { Individual } \\
\text { education on diet \& } \\
\text { medication }\end{array}$ & $\begin{array}{l}\text { Diet control and usual } \\
\text { medication delivered by } \\
\text { dietician }\end{array}$ & 6 & c \\
\hline
\end{tabular}


Table 1 (Continued)

\begin{tabular}{|c|c|c|c|c|c|c|c|c|c|c|c|}
\hline $\begin{array}{l}\text { Year, country, } \\
\text { reference }\end{array}$ & $\begin{array}{l}\text { Number of } \\
\text { participants } \\
\text { recruited/at } \\
\text { follow-up }\end{array}$ & $\begin{array}{l}\text { Mean } \\
\text { age } \\
\text { (S.D. or } \\
\text { range) } \\
\text { (years) }\end{array}$ & $\begin{array}{l}\text { Mean (S.D.) } \\
\% \text { glycated } \\
\text { haemoglo- } \\
\text { bin at base- } \\
\text { line }\end{array}$ & $\begin{array}{l}\text { Clinical sub- } \\
\text { group (type of } \\
\text { treatment) }\end{array}$ & $\begin{array}{l}\text { Mean (S.D. } \\
\text { or range) } \\
\text { duration of } \\
\text { diabetes } \\
\text { (years) }\end{array}$ & $\begin{array}{l}\text { Model and duration } \\
\text { of therapy in inter- } \\
\text { vention group }\end{array}$ & $\begin{array}{l}\text { Regimen in } \\
\text { intervention group and } \\
\text { speciality of therapist }\end{array}$ & $\begin{array}{l}\text { Model and duration } \\
\text { of therapy in } \\
\text { control group }\end{array}$ & $\begin{array}{l}\text { Regimen in control group } \\
\text { and speciality of therapist }\end{array}$ & $\begin{array}{l}\text { Follow-up } \\
\text { months }\end{array}$ & Quality \\
\hline $\begin{array}{l}\text { 2004, UK, } \\
\text { Clark }\end{array}$ & $100 / 94$ & $\begin{array}{l}59.5 \\
(40- \\
70)\end{array}$ & $8.4(1.6)$ & $\begin{array}{l}\text { Sub-optimum } \\
\text { glycaemic } \\
\text { control (D, T, I) }\end{array}$ & $8.0(\mathrm{NS})$ & $\begin{array}{l}\text { Individual CBT for } 7 \\
\text { weeks }\end{array}$ & $\begin{array}{l}4 \text { Motivational } \\
\text { interviewing sessions } \\
\text { and goal setting by } \\
\text { psychologist }\end{array}$ & $\begin{array}{l}\text { Usual care; not } \\
\text { described }\end{array}$ & $\begin{array}{l}\text { Usual diabetes care; } \\
\text { speciality not specified }\end{array}$ & 3 & B \\
\hline 2004, UK, Kirk & $70 / 59$ & $\begin{array}{l}57.6 \\
( \pm 7.9)\end{array}$ & 8.7 (NS) & $\begin{array}{l}\text { Sedentary } \\
(\mathrm{D}, \mathrm{T}, \mathrm{I})\end{array}$ & $6.0(\mathrm{NS})$ & $\begin{array}{l}\text { Individual CBT for } \\
36 \text { weeks }\end{array}$ & $\begin{array}{l}2 \text { ( } 30 \mathrm{~min}) \text { face-to-face } \\
\text { consultations } \& 4 \\
\text { telephone sessions by a } \\
\text { trained exercise science } \\
\text { graduate (research } \\
\text { assistant: single } \\
\text { format) }\end{array}$ & $\begin{array}{l}\text { Usual care and } \\
\text { standard DUK } \\
\text { leaflets }\end{array}$ & $\begin{array}{l}\text { Regimen not described; } \\
\text { speciality not specified }\end{array}$ & 6 & c \\
\hline $\begin{array}{l}\text { 2004, China, } \\
\text { Huang }\end{array}$ & $120 / 105$ & $\begin{array}{l}57 \\
( \pm 8.5)\end{array}$ & $\begin{array}{l}10.6 \\
( \pm 1.47)\end{array}$ & $\begin{array}{l}\text { General with } \\
\text { no somatic/ } \\
\text { mental } \\
\text { disorders } \\
(D, T, I)\end{array}$ & $5.75( \pm 2.2)$ & $\begin{array}{l}\text { Individual CBT and } \\
\text { counselling and } \\
\text { group education for } \\
24 \text { weeks }\end{array}$ & $\begin{array}{l}12 \text { mixed sessions of } \\
\text { health education (in } \\
\text { groups) and individual } \\
\text { psychotherapy and } \\
\text { counselling by } \\
\text { psychotherapist and } \\
\text { clinical therapist }\end{array}$ & $\begin{array}{l}\text { Usual care and } \\
\text { medication for } 6 \\
\text { months, not } \\
\text { described }\end{array}$ & $\begin{array}{l}\text { Individual diet and exercise } \\
\text { advice; speciality not } \\
\text { specified }\end{array}$ & 6 & c \\
\hline $\begin{array}{l}\text { 2004, USA, } \\
\text { Whittemore }\end{array}$ & $53 / 49$ & $\begin{array}{l}57.6 \\
( \pm 10.9)\end{array}$ & $7.7( \pm 1.3)$ & $\begin{array}{l}\text { Women with } \\
\text { no end stage } \\
\text { disease }(\mathrm{D}, \mathrm{T}, \mathrm{I})\end{array}$ & $2.7( \pm 3.0)$ & $\begin{array}{l}\text { Individual CBT and } \\
\text { education for } 6 \\
\text { months }\end{array}$ & $\begin{array}{l}6 \text { nurse-coaching } \\
\text { sessions \& } 2 \text { telephone } \\
\text { support sessions over } 6 \\
\text { months by registered } \\
\text { nurse }\end{array}$ & $\begin{array}{l}\text { Usual care on } \\
\text { waiting list (single } \\
\text { format) }\end{array}$ & $\begin{array}{l}\text { Regular appointments with } \\
\text { primary care providers at } \\
\text { 3-4-month intervals; } \\
\text { nurses, internists, family } \\
\text { practice specialist \& } \\
\text { endocrinologist }\end{array}$ & 6 & c \\
\hline $\begin{array}{l}\text { 2005, China, } \\
\text { Bing }\end{array}$ & $300 / 300$ & $\begin{array}{l}52.85 \\
( \pm 17.2)\end{array}$ & $9.6( \pm 1.75)$ & General (D, T, I) & $5.0( \pm 3.63)$ & $\begin{array}{l}\text { Group education } \\
\text { (patients \& } \\
\text { families) and } \\
\text { individual CBT for } 6 \\
\text { months. }\end{array}$ & $\begin{array}{l}24 \text { individual } \\
\text { relaxation sessions } \\
\text { over } 6 \text { months and } 24 \\
\text { educational sessions } \\
\text { over } 6 \text { months with } \\
\text { doctor, nurse and } \\
\text { dieticians }\end{array}$ & $\begin{array}{l}\text { Usual care and } \\
\text { medication for } 6 \\
\text { months (single } \\
\text { format) }\end{array}$ & $\begin{array}{l}\text { Conventional diabetes care; } \\
\text { speciality not specified }\end{array}$ & 6 & c \\
\hline $\begin{array}{l}\text { 2005, China, } \\
\text { Zhao }\end{array}$ & $80 / 80$ & $\begin{array}{l}67.3 \\
( \pm 4.53)\end{array}$ & NS & $\begin{array}{l}\text { General (D, T, I) } \\
\text { with 2nd \& 3rd } \\
\text { degree diabetic } \\
\text { retinopathy }\end{array}$ & NS & $\begin{array}{l}\text { Individual } \mathrm{CBT} \text { for } \\
28 \text { days }\end{array}$ & $\begin{array}{l}8 \text { telephone } \\
\text { consultations over } 28 \\
\text { days and patients to } \\
\text { utilise relaxation } \\
\text { techniques twice a day } \\
(2 \text { min } \times 30 \text { min }) \text { for } 28 \\
\text { days. Speciality not } \\
\text { specified }\end{array}$ & $\begin{array}{l}\text { Basic regular } \\
\text { medical treatment, } \\
\text { not described } \\
\text { (single format) }\end{array}$ & $\begin{array}{l}\text { Conventional diabetes care; } \\
\text { speciality not specified }\end{array}$ & 1 & c \\
\hline $\begin{array}{l}\text { 2005, USA, } \\
\text { McGinnis }\end{array}$ & $39 / 30$ & $\begin{array}{l}51.5 \\
\text { (NS) }\end{array}$ & 7.2 (NS) & General (D, T, I) & NS & $\begin{array}{l}\text { Individual CBT for } \\
10 \text { weeks }\end{array}$ & $\begin{array}{l}10 \text { sessions of bio- } \\
\text { feedback \& relaxation. } \\
\text { Speciality not specified }\end{array}$ & $\begin{array}{l}\text { One-to-one } \\
\text { diabetes education } \\
\text { sessions with } \\
\text { leaflets provided by } \\
\text { registered nurse }\end{array}$ & $\begin{array}{l}3 \text { ( } 60-75 \text { min each) } \\
\text { sessions held } 3 / 4 \text { weeks } \\
\text { apart; speciality not } \\
\text { specified }\end{array}$ & 2 & c \\
\hline $\begin{array}{l}\text { 2006, } \\
\text { Germany, } \\
\text { Siebolds }\end{array}$ & $250 / 223$ & $\begin{array}{l}59.6 \\
(45- \\
70)\end{array}$ & 8.4 (NS) & General $(\mathrm{D}, \mathrm{T})$ & 5.33 (NS) & $\begin{array}{l}\text { Individual } \\
\text { standardised } \\
\text { counselling and the } \\
\text { use of food and } \\
\text { blood glucose } \\
\text { diaries }\end{array}$ & $\begin{array}{l}\text { Four individual } \\
\text { counselling sessions } \\
\text { provided by physician } \\
\text { and nurse over } 24 \\
\text { weeks counselling } \\
\text { algorithm focussed on } \\
\text { self-perception, self- } \\
\text { reflection and self- } \\
\text { regulation }\end{array}$ & $\begin{array}{l}\text { Individual non- } \\
\text { standardised } \\
\text { advice on diet and } \\
\text { lifestyle. }\end{array}$ & $\begin{array}{l}\text { Speciality not specified, } \\
\text { details of advice not } \\
\text { specified, spread over } 4 \\
\text { sessions in } 24 \text { weeks. }\end{array}$ & 6 & c \\
\hline
\end{tabular}




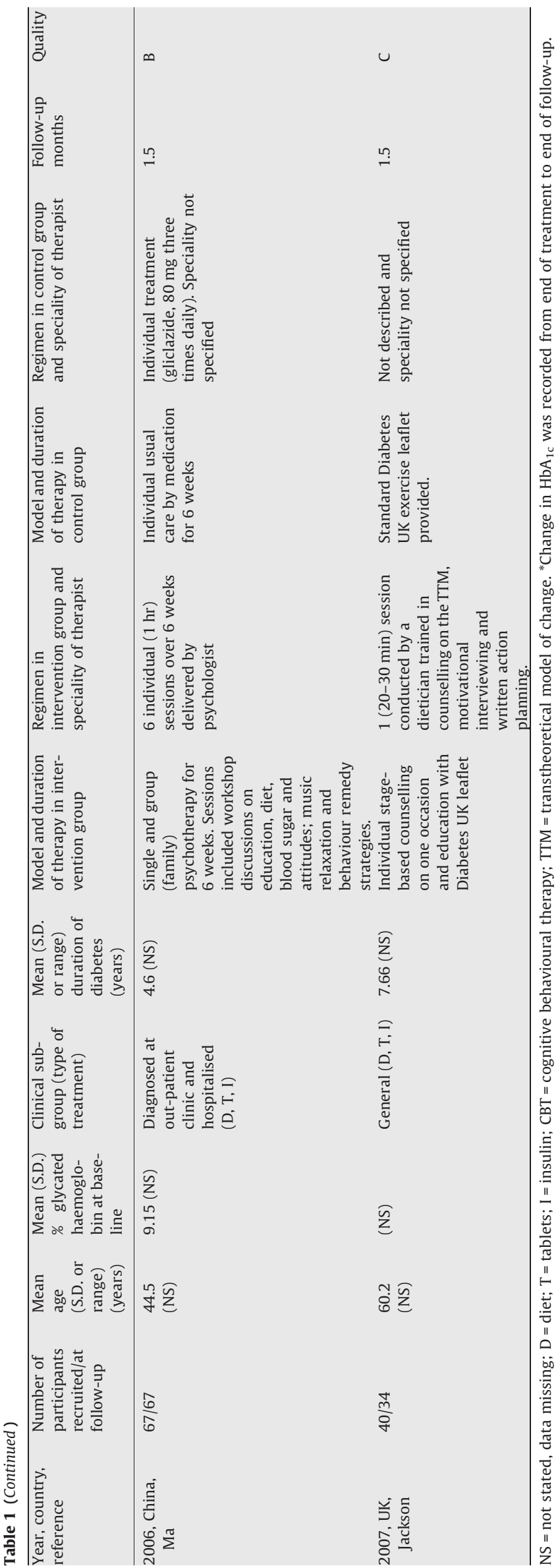

\subsubsection{Participants}

The mean time since diagnosis was 5.3 (S.D. 2.94) years and the mean sample size was 105.3 (S.D. 85.59).

\subsubsection{Follow-up}

The mean follow-up of interventions was 18.0 (S.D. 10.30) weeks.

These additional 10 trials were pooled with the previous 25 trials from the original review [2] resulting in a total of 35 trials (Table 1). Reasons for exclusion are shown in Fig. 1.

\subsection{Description of included trials from original and new review}

\subsubsection{Sample size}

The mean sample size of the 10 new trials was higher (105.3, S.D. 88.15) than the previous review (47.6, S.D. 27.5) and added 1053 (47\%) more patients to the original 1190 patients (53\%) to provide 2243 patients in total.

\subsubsection{Study quality}

Of the 35 trials one study was classified A for quality [31], 8 studies were classified as B [21,32-38] and the remaining 26 studies were classified C [22-31,39-55]. Four studies reported analysing the results by intention to treat $[21,25,31,38]$.

\subsubsection{Psychological therapies used}

The majority of studies examined cognitive behaviour therapy $[25,26,31,35-38,41,43,49,54]$ including strategies of relaxation [21-24,28,34,48,50,53-55], problem solving [26,42], contract setting $[40,46]$, goal setting [52], self-monitoring of behaviours $[32,39]$ and enlisting social support [44]. Seven studies used counselling techniques $[27,30,32,33,45,47,51]$. Four studies compared a more intensive psychological therapy with a control that was less intensive $[32,37,44,51]$.

\subsubsection{Intervention delivery specialist}

Sixteen of the 35 trials were delivered by psychological specialists $[21,24,27,31,33,37,38,40,42,43,47-51,53], 13$ were delivered by generalists $[23,25,26,30,31,35,36,39,41,44,45,52,55]$ and 6 did not report the specialist $[22,28,32,34,46,54]$.

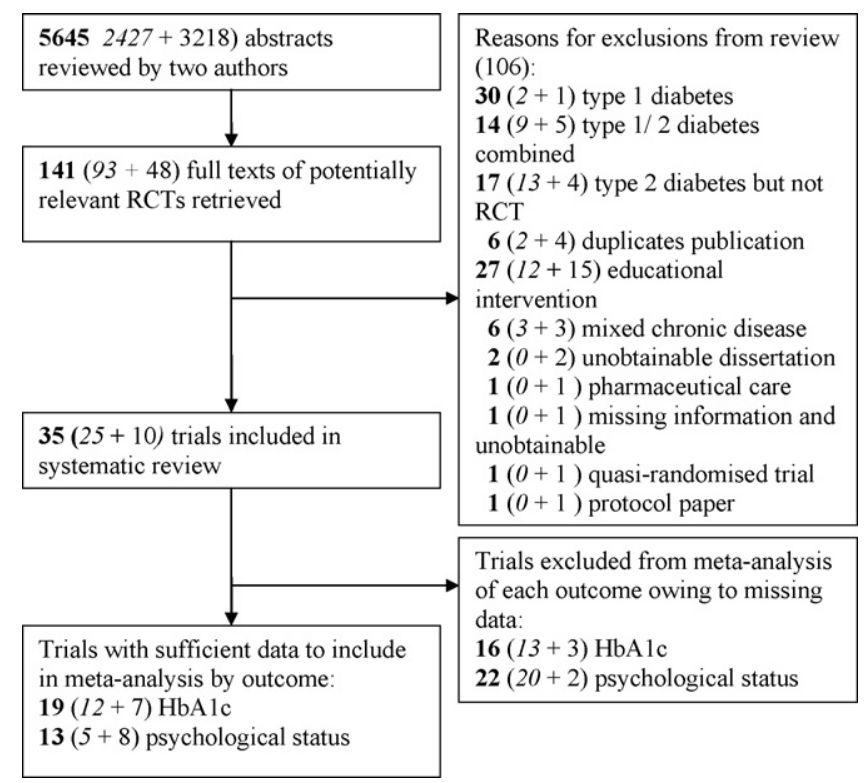

Fig. 1. QUORUM flow chart. 
Random-effects meta-analysis of psychological interventions on glycaemic control

Study (year)
Wing (1991)
D'ermao (1992)
Lane (1993)
Campbell (1996)
Aikens (1997)
Henry (1997)
Lustman (1998)
Ridgeway (1999)
Huang (2001)
Kenardy (2002)
Tsujiuchi (2002)
Zeng (2003)
Clark (2004)
Kirk (2004)
Huang (2004)
Whittemore (2004)
Bing (2005)
Siebolds (2006)
Ma (2006)
Total

Standardised Mean diff. $(95 \% \mathrm{Cl}) \quad$ \% Weight

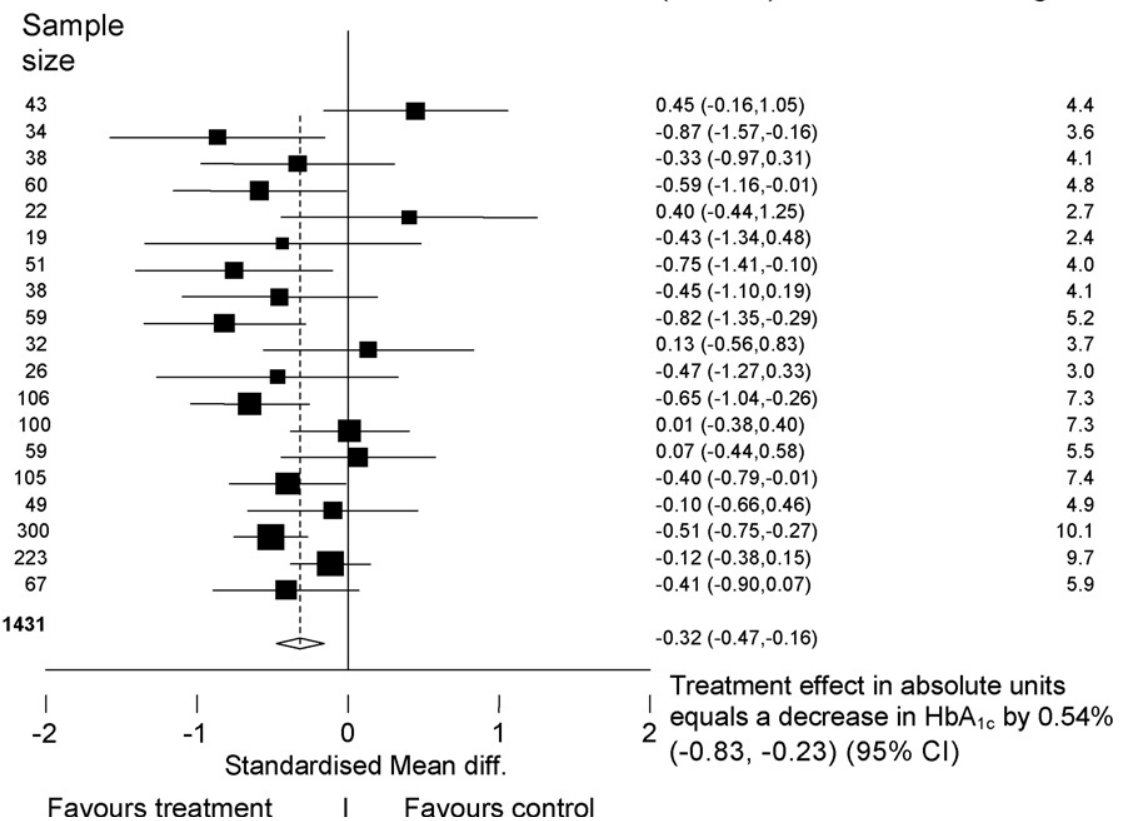

Fig. 2. Meta-analysis illustrating the standardised effects of psychological interventions on glycaemic control.

\subsubsection{Intervention format}

The mean duration of interventions was 13.7 (S.D. 11.06) weeks. Fourteen trials used a group format [31,33,36,37,39$42,44,48,49,51,54,55], \quad 16$ used a single format [21,22,25$28,30,31,34,35,38,43,46,50,52,53]$ and 5 used a combination $[21,24,32,45,47]$. Eleven of the 35 trials used an educational component in the intervention in addition to the psychological component $[21,23,24,26,27,30,31,36,43,45,54]$.

\subsubsection{Follow-up}

The mean follow-up of interventions was 18.5 (S.D. 10.4) weeks.

\subsubsection{Study location}

Only 3 trials were UK-based $[25,30,38]$ and 17 were undertaken in USA [26,28,31,32,34,36,40-42,44-48,50,52,54], 7 in China [2124,27,51,53], 4 in Australia [33,35,39,49], 1 in Canada [39], 1 in Germany [31], 1 in Japan [55] and 1 in Spain [43]. Eight studies were translated into English (one was Spanish [43] and seven were Chinese [21-24,27,51,53]).

\subsubsection{Intervention fidelity}

The precise training the generalist HCPs received in delivering the psychological interventions was not described for the majority of studies. Only one of the trials delivered by the generalists described the training received [30]. None of the 35 trials reported quality assessment procedures to ensure intervention fidelity.

\subsection{Meta-analysis}

\subsubsection{Glycaemic control}

Seven new trials reported $\mathrm{HbA}_{1 \mathrm{c}}$ values as their primary outcome bringing the total to 20 . One study was excluded from the meta-analysis due to missing data [28]. Sixteen trials reported baseline $\mathrm{HbA}_{1 \mathrm{c}}$ values for intervention and control arms at $9.53 \%$ (S.D. 1.59) and $9.66 \%$ (S.D. 1.67) respectively and three studies reported the mean difference between baseline and follow-up.

In total, 19 studies were meta-analysed, 14 of which reported improvements in $\mathrm{HbA}_{1 \mathrm{c}}$, 4 reported deterioration and 1 showed no difference (Fig. 2). With the random effects model, the pooled effect size for $\mathrm{HbA}_{1 \mathrm{c}}$ was -0.32 (95\% CI: -0.47 to -0.16 ). The treatment effect in absolute units was a decrease of $0.54 \%$ ( -0.83 to -0.23 ) in $\mathrm{HbA}_{1 \mathrm{c}}$ indicating an improvement in glycaemic control.

The $\chi^{2}$ test for heterogeneity was significant $\left(\chi^{2}=32.29\right.$; $\mathrm{df}=18 ; p=0.020)$ and $I^{2}$ test indicates below moderate heterogeneity (44\%). There was no evidence of publication bias in the funnel plot (Fig. 3), the Egger's test or the Begg adjusted rank correlation test ( $p=0.438$ and $p=0.400$ respectively).

There was no statistical association between $\mathrm{HbA}_{1 \mathrm{c}}$ and duration of follow-up (regression coefficient $(\beta)=0.01$ $($ S.E. $=0.01), p=0.275$ ) or duration of therapy (regression coefficient $=-0.009$ (S.E. $=0.01), p=0.488$ ). However there was some evidence of an association between improvements in $\mathrm{HbA}_{1 \mathrm{c}}$ and increased number of sessions (regression coefficient $=-0.04$ $($ S.E. $=0.01), p=0.001$ ).

In sensitivity analysis, the exclusion of two studies that used less intensive psychological therapy as the control intervention,

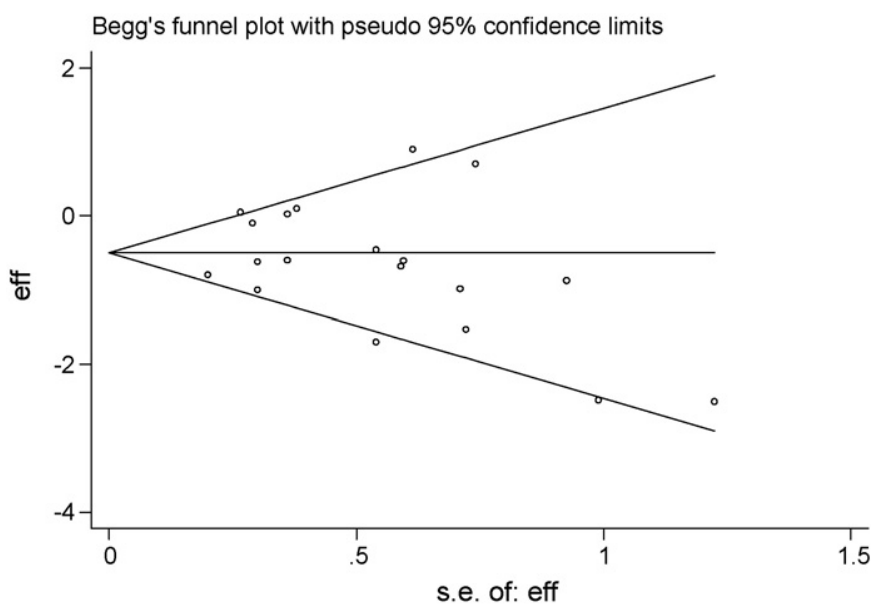

Fig. 3. Funnel plot illustrating publication bias of psychological interventions on glycaemic control. 


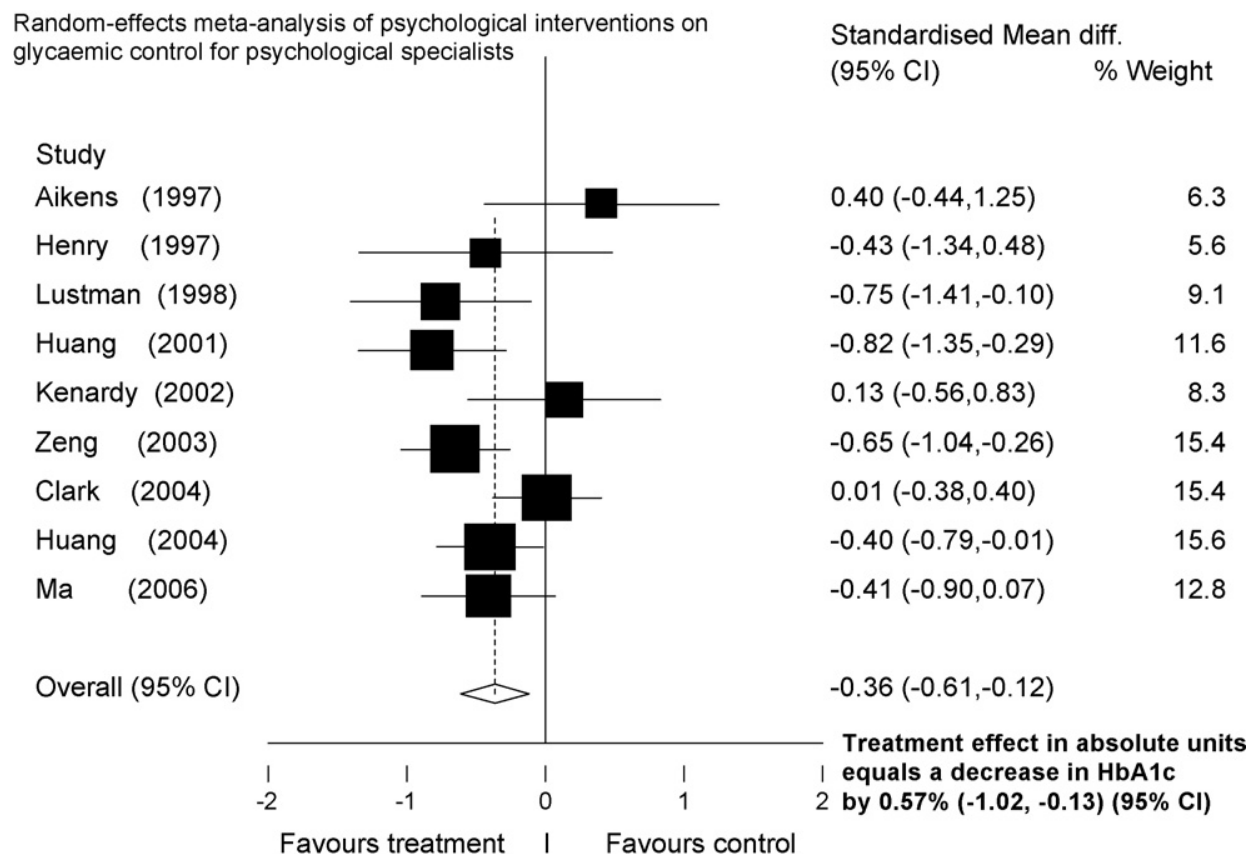

Fig. 4. Meta-analysis illustrating the standardised effects of psychological specialists delivering psychological interventions on glycaemic control.

resulted in a larger estimate of the pooled effect size $(-0.54[95 \%$ CI: -0.84 to -0.23$]$ ) equating to an absolute decrease of $0.65 \%$ $(-0.94$ to -0.36$)$ in $\mathrm{HbA}_{1 \mathrm{c}}$.

\subsubsection{Psychotherapist/psychologist and generalists}

Psychological specialists and generalist clinicians were involved in the delivery of the interventions and meta-analyses were carried out on these two sub-groups.

The $\chi^{2}$ test for the psychological specialists showed evidence of heterogeneity $\left(\chi^{2}=14.92 ; \mathrm{df}=8 ; p=0.061\right)$, however the $I^{2}$ test illustrates below moderate heterogeneity (46\%). Using the random effects model, the pooled standardised effect size estimate for $\mathrm{HbA}_{1 \mathrm{c}}$ was -0.36 ( -0.61 to -0.12 ) (Fig. 4 ).
The $\chi^{2}$ test for the generalist clinicians showed evidence of heterogeneity $\left(\chi^{2}=16.77 ; \mathrm{df}=8 ; p=0.033\right)$ and the $I^{2}$ test illustrated moderate risk of heterogeneity (52\%). Using the random effects model, the pooled standardised effect size estimate for $\mathrm{HbA}_{1 \mathrm{c}}$ was -0.36 ( -0.61 to -0.12 ) (Fig. 5).

The treatment effect in absolute units was a decrease of $0.57 \%$ $(-1.02$ to -0.13$)$ in $\mathrm{HbA}_{1 \mathrm{c}}$ for the psychological specialists and a decrease of $0.51 \%(-1.00$ to -0.01$)$ in $\mathrm{HbA}_{1 \mathrm{c}}$ for the generalist clinicians.

\subsubsection{Psychological status}

Five new trials reported measures of psychological status as an outcome and these were included in the meta-analysis. Of these 13

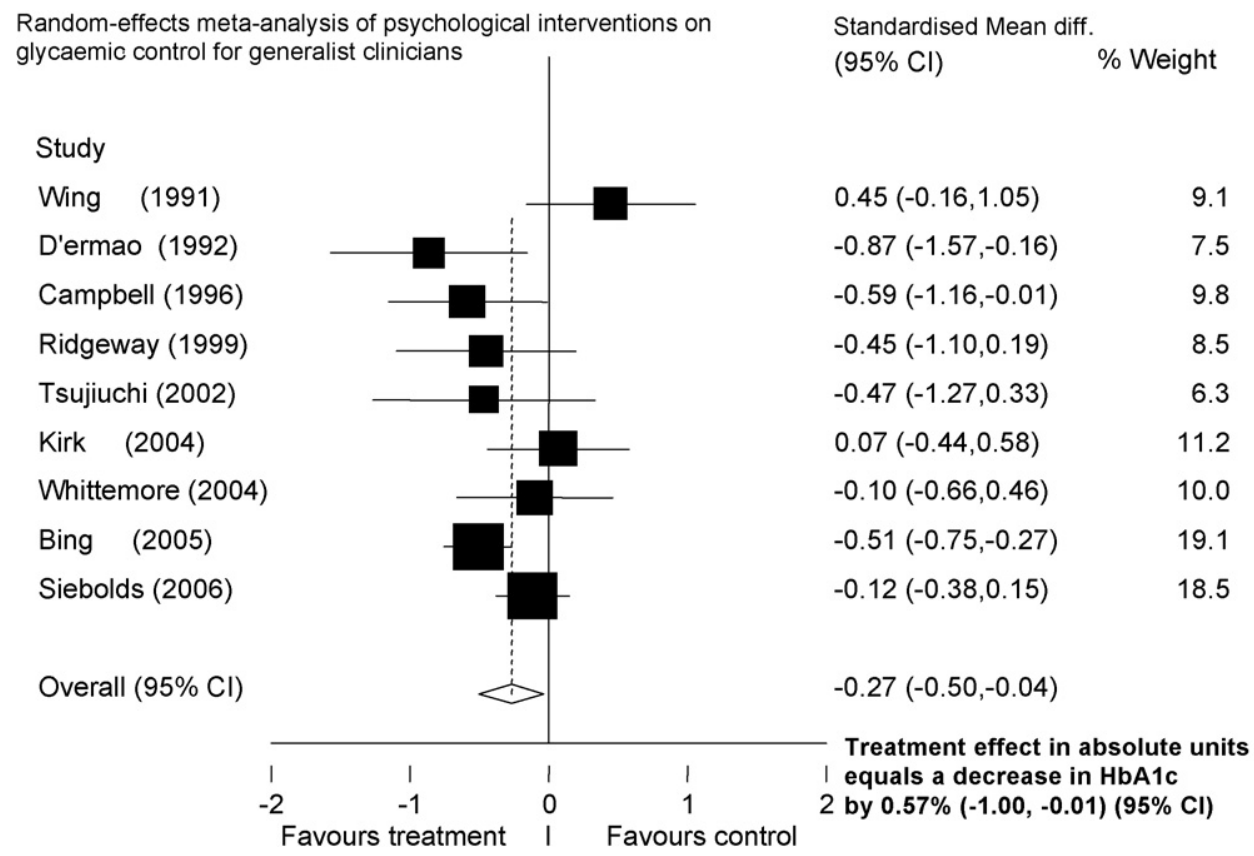

Fig. 5. Meta-analysis illustrating the standardised effects of generalist clinicians delivering psychological interventions on glycaemic control.

Please cite this article in press as: Alam R, et al. An updated meta-analysis to assess the effectiveness of psychological interventions delivered by psychological specialists and generalist clinicians on glycaemic control and on psychological status. Patient Educ Couns (2008), doi:10.1016/j.pec.2008.08.026 


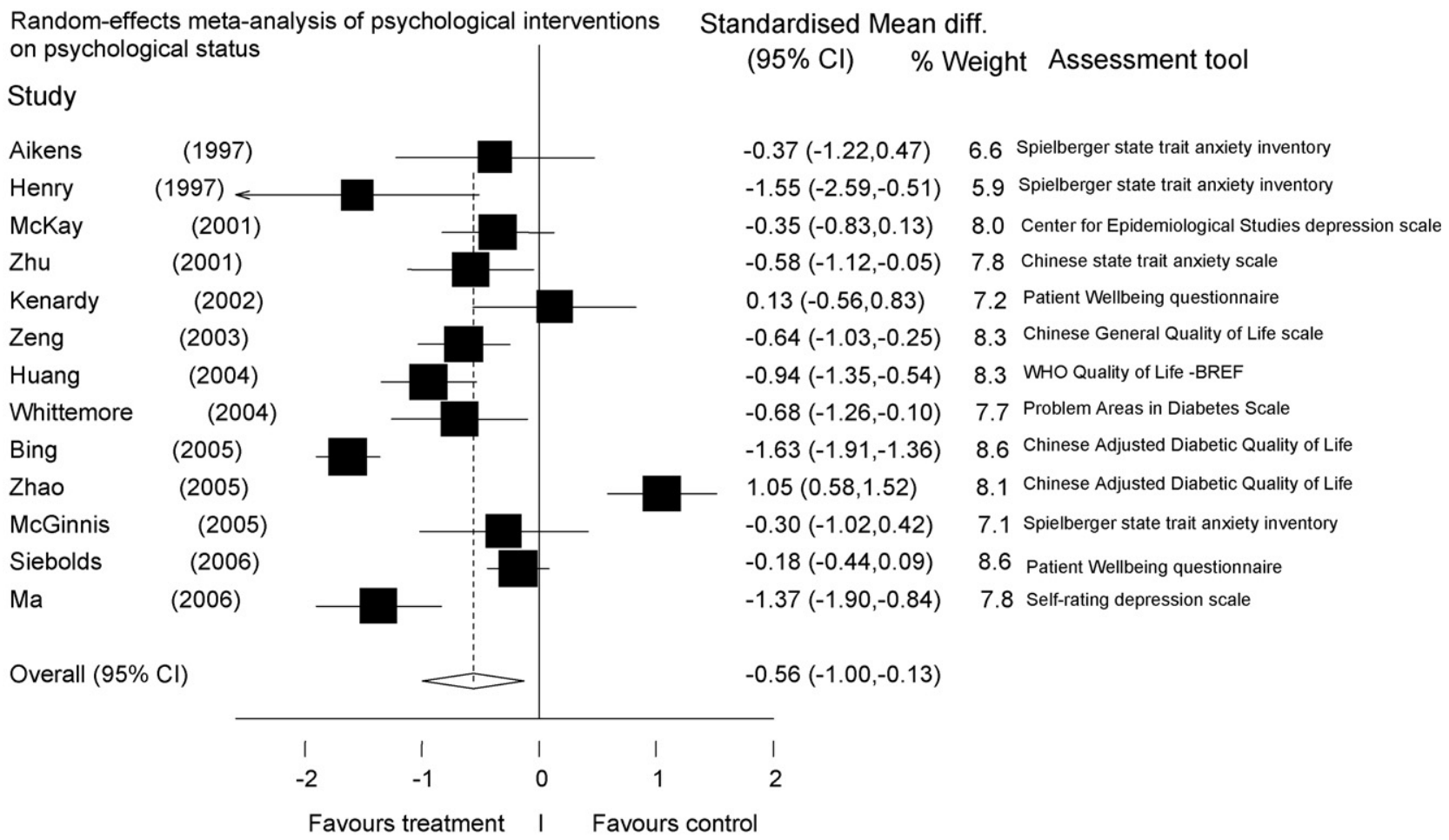

Fig. 6. Meta-analysis of standardised change scores in psychological status.

trials in total, 7 were delivered by psychological specialists, 4 were delivered by generalist clinicians which prevented a sub-group analysis by delivery specialist. Two trials did not report the specialist.

The test of heterogeneity from the random effects model indicates that the studies are statistically different $\left(\chi^{2}\right.$ test of heterogeneity $=132.45 ; \quad \mathrm{df}=12 ; p<0.001)$. With the random effects model, the pooled effect size effect for psychological distress was -0.56 (95\% CI: -1.00 to -0.13 ) (Fig. 6). The weighted mean difference was a decrease of 7.9 units $(-12.7$ to -3.15$)$ in psychological status.

There was no publication bias (plot not shown; Begg's test: $p=0.714$ ). There was evidence of statistical association between improvements in psychological status and increased duration of therapy (regression coefficient $=-0.43$ (S.E. $=0.03$ ), $p<0.001$ ), increased follow-up length (regression coefficient $=0.52$ (S.E. $=$ $0.08), p<0.001$ ) and increased number of sessions (regression coefficient $=0.15($ S.E. $=0.14), p<0.001$ ) with the exception of one trial [22].

\section{Discussion and conclusion}

\subsection{Discussion}

The current review identified 10 additional trials to add to the original review and in total 35 RCTs were included in the review. Meta-analysis of 19 trials of psychological interventions demonstrated a $0.54 \%$ reduction in $\mathrm{HbA}_{1 \mathrm{c}}$. The psychological specialists delivered a $0.57 \%$ reduction in $\mathrm{HbA}_{1 \mathrm{c}}$ and the generalist clinicians a $0.51 \%$ reduction. The positive trend in increasing sample size was not concomitant with improvements in the reporting of potential biases and hence trial quality, which remained poor.

\subsubsection{Strengths and weaknesses of the study}

Permission from the original reviewers to use their Cochrane review protocol and the involvement of the original reviewers was a study strength enabling consistency between the review procedures.

The level of heterogeneity was less than ideal in particular relation to the interventions and the degree of intervention fidelity but was satisfactory in relation to the outcome of $\mathrm{HbA}_{1 \mathrm{c}}$ and trial design. Pooled data from 13 trials measuring psychological distress reported a significant reduction in psychological distress in the intervention arms, however, nine different psychological measurement tools were used, three of which were Chinese. The variability in the tools and scales used to measure psychological distress between the studies are likely to introduce some methodological heterogeneity and the lack of a consistent scale between studies requires the reader to interpret these units with caution.

\subsubsection{Strengths and weaknesses in relation to other studies}

Our meta-analysis of trials assessing glycaemic control using measures of $\mathrm{HbA}_{1 \mathrm{c}}$ found a smaller effect size compared to the $1 \%$ reduction reported in the original review [2]. Consistent with the previous review [2], however, we did observe improvements in the overall effect ( $0.65 \%$ reduction) when two studies using a less intensive psychological therapy as controls were excluded [37,44]. Trial quality has not improved with later studies and therefore cannot account for the observed reduction in effect. It is possible that an increase in clinician consciousness regarding the impact of psychological factors on patients, may have resulted in greater levels of psychological care being experienced in the comparison groups of newer trials, thus partially accounting for the reduced effect size observed in our update. Additionally, the newer trials tended to be larger in sample size, longer in duration and follow-up and their smaller effect size had a more profound impact on the overall pooled difference.

Furthermore, we report a statistically significant association between effect size and the number of sessions in the intervention. It appears that features of intervention and trial design such as length of follow-up and duration of intervention may not be as important as the number of sessions provided. The association 
between improved $\mathrm{HbA}_{1 \mathrm{c}}$ and an increase in the number of sessions is possibly related to repeated exposures providing ongoing social support resulting in enhanced patient concordance and outcomes [56].

The sub-group analysis of psychological specialists and generalist clinicians indicates that generalists may be similarly effective in delivering psychological interventions in reducing $\mathrm{HbA}_{1 \mathrm{c}}$. This supports an earlier finding by Mojica et al. [57] reporting on the effectiveness of different health care providers, including generalist and psychological specialist, on smoking cessation interventions. Only one trial in our review reported the specific training received by the generalists [30], however we believe that the success achieved by the generalists may be attributed to the acquisition of additional knowledge, training and skills. The inconsistency in reporting the specific training received in most trials suggests the need for the inclusion of such data in future studies.

Nonetheless, the effectiveness observed here contributes to previous reviews suggesting that people with diabetes could benefit from receiving care from generalist clinicians and diabetes specialist professionals who have received training in delivering psychological care [58].

\subsection{Conclusion}

The reductions observed in $\mathrm{HbA}_{1 \mathrm{c}}$ are clinically significant in reducing microvascular and macrovascular complications [1]. Furthermore, there have been previous concerns regarding the potential benefits of psychological care in patients with longstanding poor glycaemic control [59] and our findings suggest that patients with long-standing sub-optimal control may benefit from psychological interventions.

The benefits of delivering psychological interventions for people with type 2 diabetes by generalist clinicians, with additional and as yet unspecified training, have been highlighted and this has the potential to make this care more widely available.

\subsection{Future recommendations}

To assess the degree of effectiveness between psychological care specialists and generalist clinicians there is a need to test differences in high quality fully powered randomised control trials in UK, including more studies delivered by generalists.

Only three of the trials in this review were conducted in UK and as such the generalisability of the findings should be interpreted with caution. Additionally, in future studies there is a need to describe the training provided to intervention delivery staff and recommendations are made to quality assure interventions to both understand and assess intervention fidelity by clinician and patient.

The use of multiple measures of psychological status introduced methodological heterogeneity into the review making interpretation of findings difficult. Researchers are urged to consider these issues in their design of RCTs and also to consider further work along the lines of Herrmans et al. [60] to compare the use and outcomes of different instruments in the same populations.

\subsection{Practice implications}

Psychological interventions appear to be effective in improving psychological distress and glycaemic control, however there is a shortage of psychological specialists within the NHS and psychological treatments are difficult to access for most patients living with diabetes.
The findings of this study suggest that with some additional training, diabetes and generalist clinicians have the potential to effectively deliver psychological interventions and improve patient outcomes. This implies that psychological care can be made more widely available to patients across health services if they do not have to depend on a service of psychological specialists in limited supply. This study supports the recommendations of both UK and international health policy (International Diabetes Federation) for the need to provide generalist clinicians with new skills in this area [9].

\section{Acknowledgements}

We would like to thank the following colleagues for their help in undertaking this review and preparing this manuscript, Chris Bridle, Khalida Ismail, Hilary Hearnshaw, Hugh McGuire and Chiu Wong.

Contributors: KI and KW developed the protocol and search strategy, JS devised and designed the study. JS, RA and KW undertook data-extraction. RL \& RA undertook the analysis. RA, RL \& JS co-wrote the initial drafts. All authors contributed to the final manuscript.

Funding: This study was funded by a grant from The Medical Research Council and JS had a post-doctoral research fellowship from the UK Department of Health NCCRCD scheme.

Competing interests: None declared.

Ethical approval: Not required.

Provenance and peer review: Not commissioned; externally peer reviewed. I confirm all patient/personal identifiers have been removed or disguised so the patient/person(s) described are not identifiable and cannot be identified through the details of the story.

\section{References}

[1] Stratton IM, Adler AI, Neil HA, Matthews DR, Manley SE, Cull CA, Hadden D, Turner RC, Holman RR. Association of glycaemia with macrovascular and microvascular complications of type 2 diabetes (ukpds 35): prospective observational study. Brit Med J 2000;321:405-12.

[2] Ismail K, Winkley K, Rabe-Hesketh S. Systematic review and meta-analysis of randomised controlled trials of psychological interventions to improve glycaemic control in patients with type 2 diabetes. Lancet 2004;363:1589-97.

[3] Gavard JA, Lustman PJ, Clouse RE. Prevalence of depression in adults with diabetes: an epidemiological evaluation. Diabetes Care 1993;16:1167-78.

[4] Lustman PJ, Griffith LS, Freedland KE, Clouse RE. The course of major depression in diabetes. Gen Hosp Psychiat 1997;19:138-43.

[5] Herpertz S, Albus C, Lichtblau K, Kohle K, Mann K, Senf W. Relationship of weight and eating disorders in type 2 diabetic patients: a multicenter study. Int J Eat Disorder 2000;28:68-77.

[6] Steed L, Cooke D, Newman S. A systematic review of psychosocial outcomes following education, self-management and psychological interventions in diabetes mellitus. Patient Educ Couns 2003;51:5-15.

[7] Hampson SE, Skinner TC, Hart J, Storey L, Gage H, Foxcroft D, Kimber A, Shaw K, Walker J. Effects of educational and psychosocial interventions for adolescents with diabetes mellitus: a systematic review. Health Technol Assess 2001;5:1-79.

[8] Winkley K, Landau S, Eisler I, Ismail K. Psychological interventions to improve glycaemic control in patients with type 1 diabetes: systematic review and meta-analysis of randomised controlled trials. Brit Med J 2006;333:65-8.

[9] Roberts S. Department of health, working together for better diabetes care: National Health Service; 2007. http://www.dh.gov.uk/en/Publicationsandstatistics/Publications/PublicationsPolicyAndGuidance/DH_074702

[10] Braillon A. Should we trust results of meta-analyses? Lancet 2004;364:14012. author reply 02-3.

[11] Nicolucci A, Tognoni G. Should we trust results of meta-analyses? Lancet 2004;364:1401. author reply 02-3.

[12] Moher D, Schulz KF, Altman DG. The consort statement: revised recommendations for improving the quality of reports of parallel-group randomised trials. Lancet 2001;357:1191-4.

[13] Ismail K, McGuire H, Winkley K. Psychological interventions for improving glycaemic control in patients with diabetes mellitus. (Protocol) Cochrane Database of Systematic Reviews 2001. doi: 10.1002/14651858.CD003055.pub3. Art No: CD003055.

[14] Landis JR, Koch GG. The measurement of observer agreement for categorical data. Biometrics 1977;33:159-74. 
[15] Dickersin K, Berlin JA. Meta-analysis: state-of-the-science. Epidemiol Rev 1992;14:154-76.

[16] Higgins JP, Thompson SG, Deeks JJ, Altman DG. Measuring inconsistency in meta-analyses. Brit Med J 2003;327:557-60.

[17] Schulz KF, Chalmers I, Hayes RJ, Altman DG. Empirical evidence of bias, dimensions of methodological quality associated with estimates of treatment effects in controlled trials. J Am Med Assoc 1995;273:408-12.

[18] Jadad AR, Moore RA, Carroll D, Jenkinson C, Reynolds DJ, Gavaghan DJ, McQuay HJ. Assessing the quality of reports of randomized clinical trials: is blinding necessary? Control Clin Trials 1996;17:1-12.

[19] Egger M, Davey Smith G, Schneider M, Minder C. Bias in meta-analysis detected by a simple, graphical test. Brit Med J 1997;315:629-34.

[20] Begg CB, Mazumdar M. Operating characteristics of a rank correlation test for publication bias. Biometrics 1994;50:1088-101.

[21] Ma ZW, Li MX, Wang CS. Effects of comprehensive psycho-intervention on life quality and carbohydrate metabolism in patients with type 2 diabetes mellitus. Zhongguo Linchuang Kangfu 2006;10:15-7 [in Chinese].

[22] Zhao L, Sun YJ, Liu QG, Huan MM, Wu J, Zhou L, Shen HJ, Jiang C. Intervention effects of music relaxation therapy on the quality of life in patients with diabetic retinopathy. Zhongguo Linchuang Kangfu 2005;9:50-3 [in Chinese].

[23] Bing S, Bo B, Hai-ling S. Effects of diabetes education and psychological intervention on comprehensive treatment of type 2 diabetes. Chin J Clin Psychol 2005;13:483-5 [in Chinese].

[24] Zeng Z, Ma L, Tang L, Luo G, Zhou H, Zhou L. Comprehensive intervention on diabetes mellitus. Chin Ment Health J 2003;17:253-5 [in Chinese].

[25] Kirk A, Mutrie N, Macintyre P, Fisher M. Effects of a 12-month activity counselling intervention on glycaemic control and on the status of cardiovascular risk factors in people with type 2 diabetes. Diabetologia 2004;47: 821-32.

[26] Whittemore R, Melkus GD, Sullivan A, Grey M. A nurse-coaching intervention for women with type 2 diabetes. Diabetes Educator 2004;30:795-804.

[27] Huang FL, Ye JH, Huang F. Effect of mental intervention on type 2 diabetics: randomized controlled study. Zhongguo Linchuang Kangfu 2004;8:5795-8 [in Chinese].

[28] McGinnis RA, McGrady A, Cox SA, Grower-Dowling KA. Biofeedback-assisted relaxation in type 2 diabetes. Diabetes Care 2005;28:2145-9.

[29] Siebolds M, Gaedeke O, Schwedes U. Self-monitoring of blood glucose-psychological aspects relevant to changes in hba1c in type 2 diabetic patients treated with diet or diet plus oral antidiabetic medication. Patient Educ Couns 2006;62:104-10.

[30] Jackson R, Asimakopoulou K, Scammell A. Assessment of the transtheoretical model as used by dietitians in promoting physical activity in people with type 2 diabetes. J Hum Nutr Diet 2007;20:27-36.

[31] Lustman PJ, Griffith LS, Freedland KE, Kissel SS, Clouse RE. Cognitive behavior therapy for depression in type 2 diabetes mellitus: a randomized, controlled trial. Ann Intern Med 1998;129:613-21.

[32] Heitzmann CA, Kaplan RM, Wilson DK, Sandler J. Sex differences in weight loss among adults with type ii diabetes mellitus. J Behav Med 1987;10:197-211.

[33] Campbell LV, Barth R, Gosper JK, Jupp JJ, Simons LA, Chisholm DJ. Impact of intensive educational approach to dietary change in NIDDM. Diabetes Care 1990; 13:841-7.

[34] Lane JD, McCaskill CC, Ross SL, Feinglos MN, Surwit RS. Relaxation training for NIDDM: predicting who may benefit. Diabetes Care 1993;16:1087-94.

[35] Campbell EM, Redman S, Moffitt PS, Sanson-Fisher RW. The relative effectiveness of educational and behavioral instruction programs for patients with NIDDM: a randomized trial. Diabetes Educator 1996;22:379-86.

[36] Ridgeway NA, Harvill DR, Harvill LM, Falin TM, Forester GM, Gose OD. Improved control of type 2 diabetes mellitus: a practical education/behavior modification program in a primary care clinic. Southern Med J 1999;92:667-72.

[37] Kenardy J, Mensch M, Bowen K, Green B, Walton J. Group therapy for binge eating in type 2 diabetes: a randomized trial. Diabet Med 2002;19:234-9.

[38] Clark M, Hampson SE, Avery L, Simpson R. Effects of a tailored lifestyle selfmanagement intervention in patients with type 2 diabetes. Brit J Health Psych 2004;9:365-79.
[39] Rabkin SW, Boyko E, Wilson A, Streja DA. A randomized clinical trial comparing behavior modification and individual counseling in the nutritional therapy of non-insulin-dependent diabetes mellitus: comparison of the effect on blood sugar, body weight, and serum lipids. Diabetes Care 1983;6:50-6.

[40] Wing RR, Epstein LH, Nowalk MP, Koeske R, Hagg S. Behavior change, weight loss, and physiological improvements in type ii diabetic patients. J Consult Clin Psych 1985;53:111-22.

[41] Hartwell S, Kaplan R, Wallace J. Comparison of behavioral interventions for control of type ii diabetes mellitus. Behav Ther 1986;17:447-61.

[42] White N, Carnahan J, Nugent CA, Iwaoka T, Dodson MA. Management of obese patients with diabetes mellitus: comparison of advice education with group management. Diabetes Care 1986;9:490-6.

[43] Rodriguez F, Alvarez M. Modificacion de conducta y mejora en el cumplimiento del tratamiento en diabeticos tipo ii. Rev Esp Ter Comportamiento 1987;5:223-47.

[44] Wing RR, Marcus MD, Epstein LH, Jawad A. A "Family-based" approach to the treatment of obese type ii diabetic patients. J Consult Clin Psych 1991;59: 156-62.

[45] D’Eramo-Melkus GA, Wylie-Rosett J, Hagan JA. Metabolic impact of education in NIDDM. Diabetes Care 1992;15:864-9.

[46] Boehm S, Schlenk EA, Raleigh E, Ronis D. Behavioral analysis and behavioral strategies to improve self-management of type ii diabetes. Clin Nurs Res 1993;2:327-44.

[47] Smith DE, Heckemeyer CM, Kratt PP, Mason DA. Motivational interviewing to improve adherence to a behavioral weight-control program for older obese women with NIDDM: a pilot study. Diabetes Care 1997;20:52-4.

[48] Aikens JE, Kiolbasa TA, Sobel R. Psychological predictors of glycemic change with relaxation training in non-insulin-dependent diabetes mellitus. Psychother Psychosom 1997;66:302-6.

[49] Henry J, Wilson P, Bruce D, Chisholm D, Rawling P. Cognitive-behavioural stress management for patients with non-insulin dependent diabetes mellitus. Psychol Health Med 1997;2:109-18.

[50] Jablon SL, Naliboff BD, Gilmore SL, Rosenthal MJ. Effects of relaxation training on glucose tolerance and diabetic control in type ii diabetes. Appl Psychophys Biof 1997;22:155-69.

[51] Huang X, Song L, Li T. The effect of social support on type ii diabetes with depression. Chin J Clin Psychol 2001;9:187-9.

[52] McKay HG, King D, Eakin EG, Seeley JR, Glasgow RE. The diabetes network internet-based physical activity intervention: a randomized pilot study. Diabetes Care 2001;24:1328-34.

[53] Zhu X, Gong Y, Yao S. The effect of biofeedback assisted relaxation training on cytokines in patients with type ii diabetes. Chin J Clin Psychol 2001;9:170-2.

[54] Surwit RS, van Tilburg MA, Zucker N, McCaskill CC, Parekh P, Feinglos MN, Edwards CL, Williams P, Lane JD. Stress management improves long-term glycemic control in type 2 diabetes. Diabetes Care 2002;25:30-4.

[55] Tsujiuchi T, Kumano H, Yoshiuchi K, He D, Tsujiuchi Y, Kuboki T, Suematsu H, Hirao K. The effect of qi-gong relaxation exercise on the control of type 2 diabetes mellitus: a randomized controlled trial. Diabetes Care 2002;25: 241-2.

[56] Delamater AM. Improving patient adherence. Cd (Clinical Diabetes) 2006;24: 71-2.

[57] Mojica WA, Suttorp MJ, Sherman SE, Morton SC, Roth EA, Maglione MA, Rhodes SL, Shekelle PG. Smoking-cessation interventions by type of provider: a metaanalysis. Am J Prev Med 2004;26:391-401.

[58] Knight KM, Dornan T, Bundy C. The diabetes educator: trying hard, but must concentrate more on behaviour. Diabetic Med 2006;23:485-501.

[59] Snoek FJ. Rapid response: not the magic bullet. Brit Med J 2006;333(June):65. Available online: http://www.bmj.com/cgi/eletters/333/7558/65 [last accessed 23/01/2008, 2006].

[60] Hermanns N, Kulzer B, Krichbaum M, Kubiak T, Haak T. How to screen for depression and emotional problems in patients with diabetes: comparison of screening characteristics of depression questionnaires, measurement of diabetes-specific emotional problems and standard clinical assessment. Diabetologia 2006;49:469-77. 